\title{
Electrophobic interaction induced impurity clustering in metals
}

\author{
Hong-Bo Zhou ${ }^{\mathrm{a}, \mathrm{b}}$, Jin-Long Wang ${ }^{\mathrm{a}, \mathrm{b}}$, W. Jiang ${ }^{\mathrm{c}}$, Guang-Hong Lu ${ }^{\mathrm{a}, \mathrm{b}, *}$, J.A. Aguiar ${ }^{\mathrm{d}, \mathrm{e}, * *}$, and Feng Liu ${ }^{\mathrm{c}, \mathrm{f}, * * *}$ \\ ${ }^{a}$ Department of Physics, Beihang University, Beijing 100191, China \\ ${ }^{\mathrm{b}}$ Beijing Key Laboratory of Advanced Nuclear Materials and Physics, Beihang University, Beijing \\ 100191, China \\ ${ }^{\mathrm{c}}$ Department of Materials Science and Engineering, University of Utah, UT 84112, USA \\ ${ }^{\mathrm{d}}$ National Renewable Energy Laboratory, Golden, CO 84101, USA \\ ${ }^{\mathrm{e}}$ Los Alamos National Laboratory, P.O. Box 1663, Los Alamos, NM 87545, USA \\ ${ }^{\mathrm{f}}$ Collaborative Innovation Center of Quantum Matter, Beijing 100084, China
}

\begin{abstract}
We introduce the concept of electrophobic interaction, analogous to hydrophobic interaction, for describing the behavior of impurity atoms in a metal, a "solvent of electrons". We demonstrate that there exists a form of electrophobic interaction between impurities with closed electron shell structure, which governs their dissolution behavior in a metal. Using $\mathrm{He}, \mathrm{Be}$ and Ar as examples, we predict by first-principles calculations that the electrophobic interaction drives $\mathrm{He}, \mathrm{Be}$ or Ar to form a close-packed cluster with a clustering energy that follows a universal power-law scaling with the number of atoms $(N)$ dissolved in a free electron gas, as well as $\mathrm{W}$ or $\mathrm{Al}$ lattice, as $E_{\mathrm{c}} \propto\left(N^{2 / 3}-N\right)$. This new concept unifies the explanation for a series of experimental observations of close-packed inert-gas bubble formation in metals, and significantly advances our fundamental understanding and capacity to predict the solute behavior of impurities in metals, a useful contribution to be considered in future material design of metals for nuclear, metallurgical, and energy applications.
\end{abstract}

Keywords: Electrophobic interaction, Impurity clustering, Metals

\footnotetext{
*Corresponding author.

**Corresponding author.

***Corresponding author.

E-mail addresses: lgh@buaa.edu.cn (G.H. Lu); jeffery.aguiar@nrel.gov (J.A. Aguiar); fliu@eng.utah.edu (F. Liu).
} 


\section{Introduction}

Hydrophobic interaction is one of the most important concepts in understanding many natural phenomena. Water is arguably the most common solvent in life and industry for a variety of technological processes. The dissolution behavior of solutes in water is governed by their hydrophobicity. It is well known that the hydrophobic interaction between hydrophobic solutes, such as waxes and fats, resulting from their repulsion of water molecules is responsible for their segregation in water; while hydrophilic solutes, such as sugar and salt, disperse uniformly in water as they form ionic or hydrogen bonds with water molecules. Hydrophobic interaction is attractive in nature so that hydrophobic particles will segregate and form clusters spontaneously in water [1,2]. The super-hydrophobic engineering materials were designed based on hydrophobic interaction [3,4]. Hydrophobic effects also play an important role in protein macromolecular synthesis and biological stability, and have been extensively investigated [5-7].

An analogy to hydrophobicity is the concept of electrophobicity that has been used to describe whether molecules like or dislike electrons [8,9], but only in a limited context of describing the affinity for electrons amongst individual molecular groups in a chemical reaction. Hence, the possibility of an electrophobic interaction has never been discussed in this context, since hardly one would dissolve molecules in a "solvent" of electrons like dissolving molecules in water. However, it is important to realize that a metal made of a large number of free electrons, where metallic-like bonding is commonly treated by a jellium model $[10,11]$, can be approximated as a solvent of electrons. Then the dissolution of impurity atoms in metal, which is a topic of great scientific and technological significance, could possibly be related to the electrophobicity of dissolved atoms (solutes). Most importantly, a form of electrophobic interaction can exist between the "electronic solutes" in metal, analogous to the hydrophobic interaction between 
solutes in water, which fundamentally governs the dissolution behavior of impurity atoms in metals. Yet, to the best of our knowledge, the concept of "electrophobic interaction" has never been discussed before.

An atom may dislike/like electrons depending on its electron shell structure. If an atom has a closed shell, i.e. He with a closed $1 s$ shell or Be with a closed $2 s$ shell, it dislikes electrons; if an atom has an open electron shell, it likes electrons. In fact, the degree of an atom dislikes/likes electrons can be roughly quantified by its electron affinity (see Supplementary Fig. S1) [12]: those with a positive affinity dislike electrons, while those with a negative affinity like electrons. Therefore, one can naturally extend the concept of electrophobicity to atoms dissolved in an electron gas. In fact, the embedding energy of an atom in an electron gas is the basis for the welldeveloped effective medium $[13,14]$ and embedded atom computational methods [15]. Here, we

demonstrate that this extension has a significant implication in achieving a new level of fundamental understanding of impurity atoms in metals, by revealing a form of electrophobic interaction between electrophobic solutes (impurity atoms) in a solvent of "electrons" (metal), in a perfect analogy with the important hydrophobic interactions associated with water.

\section{Methods}

DFT calculations. Our calculations were performed using the pseudopotential plane-wave method as implemented in the VASP code $[16,17]$ based on the density functional theory (DFT). We used the generalized gradient approximation of Perdew and Wang [18] and projected augmented wave potentials [19], with a plane wave energy cutoff of $500 \mathrm{eV}$. The bcc supercell of 128 atoms was used and its Brillouin zone was sampled with $(3 \times 3 \times 3)$ k-points by the Monkhorst-Pack scheme [20]. The energy minimization is continued until the forces on all the 
atoms are converged to less than $10^{-3} \mathrm{eV} / \AA$. To possibly better account for dispersive interaction between the closed-shell atoms, test calculations are also done for He dimers in vacuum and electron gas with Van der Waals exchange-correlation functional, which made no significant difference.

Calculation of $\mathrm{He}$ and $\mathrm{H}$ solution energy in a homogeneous electron gas as a function of the electron density. The dissolution of $\mathrm{He}$ and $\mathrm{H}$ in a homogeneous electron gas with different electron density is simulated by adding electrons in an empty box with a compensating uniform positive charge background. First, we constructed a vacuum cubic supercell with fixed volume. Then, a given number of electrons is added for a given electron density along with the same density of uniform positive charge background. Next, $\mathrm{He} / \mathrm{H}$ is added in the supercell at different electron density, and the He and H solution energy is calculated, as shown in Fig. 1.

Calculation of the He-induced deformation energy and electron density at defect sites. In order to get the He-induced deformation energy, He is first placed at a given defect site (TIS, OIS or SS), and both the atomic positions and supercell size are relaxed by energy minimization. Then, He is removed, and the atomic positions and the supercell size are fixed to account for the deformation induced by He, namely the difference between the total energy of the distorted lattice and that of the equilibrium induced by He. The electron density at the defect site is also calculated with both the supercell size and the atomic coordinates fixed.

Irradiation Experiments and Electron Microscopy. Effects of inert-gas bubble formation in materials were performed through a series of jointly coupled irradiation and subsequent high resolution (scanning) transmission electron microscopy (S/TEM) studies. Resolving the effects of Xe clustering in metals, Al metal targets were selected. Al metal was targeted with a single $250 \mathrm{keV} \mathrm{Xe}$ ion beam at room temperature. 


\section{Results and discussion}

We first illustrate the concept of an electrophobic solute (a closed-shell impurity) vs. an electrophilic solute (an open-shell impurity) in a solvent of electrons by reviewing the solution energy (or embedding energy) of a He atom vs. a $\mathrm{H}$ atom in a free electron gas as a function of electron density calculated from first-principles (see Methods), as shown in Fig. 1. The results of Fig. 1 are well known from previous studies [21], but here we re-consider them in a new context of electrophobicity. Helium, an electrophobic solute, dislikes electrons having a positive solution energy that increases with the increasing electron density. In contrast, $\mathrm{H}$, is an electrophilic solute, likes electrons having a negative solution energy whose magnitude increases initially and then decreases with increasing electron density. The turn-around behavior for H solution energy that eventually becomes positive is because at too high an electron density, the positive electronelectron Coulomb repulsion dominates over the negative H-electron attraction. From this point onward we will therefore limit our calculations for relatively low electron density.

Next, we derive a simple analytical form to describe solute behavior in light of electrophobic interactions. We start by considering an impurity atom having a closed shell that acts as an electrophobic solute in a free-electron gas, we may approximate their repulsive interaction with the surrounding electron media using a hard-sphere potential, $V(r)=\left\{\begin{array}{l}\beta, r=r_{0} \\ 0, r \geq r_{0}\end{array}\right.$, as illustrated in Fig. 2a. If there are $N$ atoms dispersed separately in the free electron gas, their total solution energy follows the classical solution $N\left(4 \pi r_{0}^{2}\right) \beta$. Now if these $N$ atoms segregate into a closelypacked crystalline structure, as shown by previous experiments [22] (see also Fig. 2b), assuming approximately a radius $R\left(R^{3} \approx N r_{0}^{3}\right)$ (Fig. 2a) for simplicity, then the solution energy of this 
cluster is calculated as $\left(4 \pi R^{2}\right) \beta$. Thus, the energy of the cluster $\left(E_{\mathrm{c}}\right)$, i.e. effectively the "electrophobic interaction" energy follows a simple power scaling relation with the cluster size as $E_{c} \propto\left(N^{2 / 3}-N\right)$.

The repulsion between $\mathrm{He}$ and electrons (positive solution energy) gives rise to an electrophobic interaction, i.e., an effective attractive interaction between He atoms. For example, between two He atoms there is zero binding energy in vacuum, but they attain a binding energy of $-0.084 \mathrm{eV}$ at an equilibrium distance of $\sim 1.65 \AA$ in an free-electron gas with an electron density of $0.08 \mathrm{e} / \AA^{3}$. Such an electrophobic interaction leads to the clustering of He atoms in an free-electron gas. The corresponding clustering energy is calculated as $E_{n H e, e}^{c}=E_{n H e, e}^{T}-n E_{1 H e, e}^{T}+(n-1) E_{e}$, where $E_{n H e, e}^{T}$ and $n E_{1 H e, e}^{T}$ is the total energy of the supercell of electron gas with $\mathrm{n}$ and one He atoms, respectively, and $E_{e}$ is the total energy of the supercell of electron gas without He. Our first-principles calculations (see Methods) of clustering energy of He up to 6 atoms in an electron gas of $0.08 \mathrm{e} / \AA^{3}$ density directly confirms the analytical form derived above, based on the simple hard-sphere or contact-area model, as shown in Fig. 2c. Also, similarly results are obtained for Be (Fig. 2d); where the model provides an excellent fit to the calculated data for both elements.

In a metal consisting of a large number of free electrons acting effectively as a solvent of electrons, embedded positive ions [13-15] perturb locally the uniform distribution of electron density. In this sense, the solubility of an impurity atom generally depends on its electrophobicity, i.e., an electrophobic impurity dislikes (or repels) electrons, such as He, will have a positive dissolution energy in metals; while an electrophilic impurity likes (or attracts) electrons, such as $\mathrm{H}$, will have a negative dissolution energy. This is indeed shown by all previous experimental and theoretical studies. For example, it has been shown that He has a positive solution energy in 
W [23-26], Fe [25,27], V [24], Pd [25] and Mo [24,25], and $\mathrm{H}$ has a negative solution energy in $\mathrm{W}$ [28-31], Fe [29], V [29], Pd [32] and Mo [29].

In greater detail, consideration of impurity dissolution in metals the interaction between the impurity and host ions must also be taken into account. The electrophobicity however should always play a key role in governing an impurity's dissolution behavior. To illustrate this point, here we examine He dissolution in $\mathrm{W}$, as shown in Table 1. First, by a simple argument of being an electrophobic solute, He prefers to occupy where the electron density is lowest. In a bcc lattice of $\mathrm{W}$, we may consider three possible sites for $\mathrm{He}$ to sit, the tetrahedral interstitial site (TIS), octahedral interstitial site (OIS) and substitutional site (SS) (i.e. the vacancy site). Apparently, the SS site with the lowest electron density is preferred, confirmed by our DFT calculations shown in Table 1, which are $6.18 \mathrm{eV}$ (TIS), $6.39 \mathrm{eV}$ (OIS) and $1.59 \mathrm{eV}$ (SS), respectively. This is in direct contrast with electrophilic impurities, such as $\mathrm{H}$, which prefer a site of optimal electron density on the inner surface of a vacancy or void to form a complex in the material $[13,21,26,28]$, but not necessarily the maximum density because too high of a density induces large Coulombic interaction, as shown in Fig. 1.

Helium is a typical interstitial impurity in metals. Generally, He will induce lattice distortion when it occupies at an interstitial site, because its atomic size is larger than the available space provided by the given interstitial site. For example, for the case of $\mathrm{He}$ in $\mathrm{W}$, the covalent radii of He and $\mathrm{W}$ atoms are $\sim 0.49 \AA$ and $\sim 1.30 \AA$, respectively; while the initial distances between He and its first nearest neighboring W atoms are $1.77 \AA$ and $1.59 \AA$ in the bulk W, respectively. This clearly indicates He induces a lattice expansion. On the other hand, as an electrophobic solute, the dissolution of $\mathrm{He}$ at an interstitial site is also directly related to the electron density. To further shed light on the physical origin underlying the stability of $\mathrm{He}$ in $\mathrm{W}$, we may divide the 
solution energy of $\mathrm{He}$ in $\mathrm{W}$ into two parts of electronic solution energy (induced by electrophobic effect) and the deformation energy induced by He. We have calculated electron densities at the He sites (see Methods) to be $0.171 \mathrm{e} / \AA^{3}$ (TIS), $0.162 \mathrm{e} / \AA^{3}$ (OIS) and $0.026 \mathrm{e} / \AA^{3}$ (SS), respectively (see Table 1). Then, using these electron densities and Fig. 1, we can estimate the electronic solution energy by a "local density approximation (LDA)" assuming: He is embedded in a homogeneous electron gas of the density corresponding to each site, where the results are provided in Table 1. Of course, the electron density is not uniform, so alternatively we also "derived" the electronic solution energy by calculating the He-induced deformation energy. He is energetically unfavorable forming chemical bonds with $\mathrm{W}$ due to its closed-electronic shell configuration, and thus the He-induced deformation energy is largely due to the size effect (or strain energy). It is calculated from the W lattice energy difference after and before $\mathrm{He}$ introduction (see Methods), which are shown along with the so derived electronic solution energy in Table 1. Evidently, one sees that by either "LDA" or "derived" methods the electronic solution energy is mostly responsible for the total solution energy, while the He-induced deformation energy plays a lesser role (see Table 1).

As a matter of fact, it is impossible to completely separate the contribution of electrophobic interactions to the He solution energy from the elastic contributions due to the lattice strain induced by $\mathrm{He}$, because the He-induced deformation (strain) energy is associated with the electron-electron interactions as well. This processing model only provides a way to further understand the stability of He in metals.

We further analyze the electronic structure of the $\mathrm{W}-\mathrm{He} / \mathrm{H}$ system by calculating the projected electronic densities of states (DOS) for $\mathrm{He} / \mathrm{H}$ at the TIS and OIS in W. It is found that only the $d$ projected DOS of W atom closest to the He/H defects, and the $p$-projected DOS of He and the $s$ - 
projected DOS of $\mathrm{H}$ exhibit notable changes, as shown in Fig. 3. The shape of the $d$-projected DOS of W is similar to that of the p-projected DOS of He shown in Fig. 3a, indicating that there may be some hybridization between these states. Moreover, the $p$-projected DOS of He at OIS near the Fermi energy level is higher than that of He at TIS, in agreement with that the electronic solution energy (Derived) of He at OIS is larger than that of He at TIS (see Table 1). This is consistent with the interaction between the $d$-projected DOS of Fe and the p-projected DOS of $\mathrm{He}$ [33]. In contrast, $\mathrm{H}$ is energetically favorable to hybridize strongly with metal atoms due to its electrophilic property. Figure 3b shows that there will be hybridization between the $d$ projected DOS of W and the s-projected DOS of H. Further, the s-projected DOS of H at TIS is higher than that of $\mathrm{H}$ at the OIS, leading to $\mathrm{H}$ energetically prefers to occupy the TIS instead of the OIS. Note that the He-W hybridization forms 'passively' due to the close-shell structure of $\mathrm{He}$, in contrast with the H-W hybridization.

In order to investigate the effects of inserting $\mathrm{He} / \mathrm{H}$ on the electronic structure of $\mathrm{W}$, the changes in the electron density induced by $\mathrm{He} / \mathrm{H}$ at the TIS and OIS were examined, as shown in Fig. 4. It clearly illustrates the difference between the interaction of He-electron and that of $\mathrm{H}-$ electron in W. From Fig. 4a and b, it is interesting to see that the He interstitial leads to significant depletion of electron density at the local region of TIS and OIS, which indicates the interaction between $\mathrm{He}$ and electron is repulsive due to the electrophobic nature of He. This property has also been found for He in other metals, such as Fe [25,33], Mo [24], and V [24]. In contrast, the electron density increases at the $\mathrm{H}$ interstitial sites as shown in Fig. 4c and d, which indicates $\mathrm{H}$ will gain electron from the surrounding $\mathrm{W}$ atoms, owing to the electrophilicity of $\mathrm{H}$. Therefore, the electrophobic/electrophilic property of $\mathrm{He} / \mathrm{H}$ leads to the different effects on the electronic structure of $\mathrm{W}$, as well as the different dissolution behavior of $\mathrm{He}$ and $\mathrm{H}$ in W. 
Now, we demonstrate the electrophobic interaction between He atoms dissolved in a W lattice. Since there are many more interstitial sites than vacancy sites, we will use the TIS to calculate the clustering energy of He in W. For example, when two He occupy neighboring TIS', the twoatom cluster binding energy is calculated as $E_{H e-H e, W}^{c}=E_{2 H e, W}^{T}-2 E_{1 H e(T I S), W}^{T}+E_{W}$, where $E_{1 H e(T I S), W}^{T}$ and $E_{2 H e, W}^{T}$ is the total energy of the W supercell with one and two He atoms, respectively, and $E_{W}$ is the total energy of the W supercell without He. The He-He binding energy is found to be $-1.09 \mathrm{eV}$ at a distance of $\sim 1.5 \AA$, in good agreement with previous studies [23,34], indicating a strong attractive "electrophobic" interaction between He atoms in W, which is also observed between other inert-gas atoms [35]. We have calculated the He clustering energy up to 6 atoms in $\mathrm{W}$. The clustering energy of $\mathrm{He}$ in $\mathrm{W}$ is calculated as $E_{n H e, W}^{c}=E_{n H e, W}^{T}-n E_{1 H e(T I S), W}^{T}+(n-1) E_{W}$, where $E_{n H e, W}^{T}$ is the total energy of the supercell of W with $\mathrm{n} \mathrm{He}$ atoms. As an example, Fig. 5a shows five TIS He atoms, which are uniformly dispersed in a W host lattice and Fig. 5b shows a segregated five-atom He cluster in W. Most important, in Fig. 5c we find the clustering energy follows the exact same universal power-law scaling relation as in a homogeneous electron gas. This is because as we show in Table 1 the electronic solution energy contributes most to the total solution energy for He in W. The same trending behavior is found for $\mathrm{Be}$ in $\mathrm{W}$. The binding energy of $\mathrm{Be}-\mathrm{Be}$ at the most stable configuration in $\mathrm{W}$ is $-1.12 \mathrm{eV}$ with a distance of $\sim 1.9 \AA$. More importantly, the clustering energy of Be atoms shown in Fig. 5d follows the same universal power-law scaling relation. Therefore, we conclude the electrophobic interaction is a leading mechanism responsible for $\mathrm{He}$ self-trapping in $\mathrm{W}$, which is a prerequisite step for He bubble formation.

We point out that the positive affinity is a necessary but may not be a sufficient condition for 
an element to cluster in an electron gas or metal, because besides positive affinity our model also requires a hard-sphere interaction potential between the element and electron gas and between elements. Particularly, in case of large charge transfer between element and metal or strong covalent bonding of elements, the model will not be applicable. This means all the inert-gas atoms will follow. Besides He, we also show the case of Xe in Al which works as expected (Fig. 6), in agreement with the experiment (Fig. 2b). The results of Be actually came as somewhat a surprise to us, which suggests $\mathrm{Mg}$ will possibly work too. We expect more and more cases will be worked out in the future.

Furthermore, based on the present electrophobic theory, He cluster should be favored at vacancy or defect sites where electron density is lower. As a test, we have calculated the He clustering energy inside a W vacancy, in reference to the solution energy of a single He atom at TIS. Indeed, the clustering energy of He in the vacancy is found larger than that at the interstitial site. Most interestingly, the clustering energy of $\mathrm{He}$ in the vacancy also follows the scaling relation with the cluster size, $E_{\mathrm{c}} \propto\left(N^{2 / 3}-N\right)$, as our theoretical model predicts (Fig. 7).

The electrophobic interaction we reveal here is an important concept, not only of great scientific interest, but also has significant technological implications. The model unifies experimental observations of inert-gas bubble formation in metals, such as He in W [36,37], Mo [38], and $\mathrm{Fe}[39,40], \mathrm{Ne}$ in $\mathrm{Mo}[41]$, Ta [41], and $\mathrm{Al}$ [42], $\mathrm{Ar}$ in $\mathrm{Al}[43]$ and $\mathrm{Xe}$ in $\mathrm{Al}[22,42,44]$ (present experiment, Fig. 2b), with a common mechanism that they are all driven by the same electrophobic model and adopt a close-packed structure. Also, Be is seen to cluster near the surface of $\mathrm{W}$ [45], as we predict in this study. Our explanation based on the concept of electrophobic interaction is fundamental and the discovered universal scaling relation between clustering energy and cluster size is expected to be generally applicable. Furthermore, our theory 
offers a guiding principle to the material design of metals for nuclear engineering, metallurgical engineering and energy applications. For example, the nature of electrophobic interaction indicates that inert gases, such as He bubbles dislike metal. Thus, bubble migration will move to where the electron density is low, which has been seen directly in experiments of $\mathrm{He}$ implantation in oxide dispersion strengthened (ODS) metal alloys. Accordingly, we further propose a novel design for radiation-damage tolerant protective shell, as shown in Fig. 8. The design explores the use a multiple layered structure consisting of a first layer of metal alloy, as used in current reactor facing incident radiation, followed a second layer of ODS metal alloy, and a third layer of an corrosion resistant oxide coating. The thickness of each layer is yet to be optimized. In certain design the second layer of ODS metal alloy may be removed. Due to the electrophobicity of inert-gas impurity atoms and bubbles, as well as the interaction between them, the chemical potential of impurities and bubbles will continuously decrease from the first metal alloy layer to the third oxide containing layer (indicated by an arrow on the left of Fig. 8). This creates a driving force to direct the diffusion of impurities and bubbles from the first layer to the third layer, effectively reducing the amount of bubbles as well as limiting the size of the bubbles in the first radiation-facing layer to significantly mitigate the damage accumulation. In addition, the embedded oxide particles in the ODS layer will increase the nucleation density of bubbles, so as to reduce the bubble size. Similarly, a high density of bubbles are expected to nucleate and form at the interface of the ODS and oxide coating, and as such, this interface should be made very rough with a large interface contact area, as shown in Fig. 8 . When the bubble density at this interface reaches a critical limit, the oxide coating layer might peel off; or one can deliberately remove the coating layer when the interfacial bubble density reaches a sufficiently high value. Then, a new oxide layer can be coated again to collect the bubbles at the interface 
and protect the first layer. For the above reasons, the lifetime of overall radiation protection shell will be significantly increased. The practical realization of the proposed design principle remains to be decided.

Finally, we point out that the dissolution of impurities in metals plays an important role in metallurgical processes, such dissolving $\mathrm{C}$ in $\mathrm{Fe}$ to make iron and steel. In this case, $\mathrm{C}$ is a strong electrophilic impurity that likes electrons [46]. Impurity doping in metal hydrides has been used as a design strategy for improving $\mathrm{H}$ storage materials [47].

\section{Summary}

We introduce the concept of electrophobic interaction, analogous to hydrophobic interaction, for describing the behavior of impurity atoms in a metal, a "solvent of electrons". We show that the electrophobic interaction between electrophobic impurities with closed electron shell structure governs their dissolution behavior in metal. Using $\mathrm{He}, \mathrm{Be}$ and $\mathrm{Ar}$ as examples, we predict by first-principles calculations that the electrophobic interaction drives $\mathrm{He}, \mathrm{Be}$ or Ar to form a close-packed cluster with a clustering energy that follows a universal power-law scaling with the number of atoms $(N)$ dissolved in a free electron gas, as well as $\mathrm{W}$ or $\mathrm{Al}$ lattice, as $E_{\mathrm{c}} \propto$ $\left(N^{2 / 3}-N\right)$. Our findings provide a general approach to better understand dissolution of impurities in metals, such as the experimental observations of close-packed inert-gas bubble formation in metals, with significant implications in future material design for nuclear engineering, metallurgical engineering and energy applications. 


\section{Acknowledgements}

This research is supported by the National Magnetic Confinement Fusion Program with Grant No. 2013GB109002, the National Natural Science Foundation of China with Grant No. 11405006, and the China Scholarship Council with Grant No. 201506025054. G.H. Lu acknowledges the financial support from the National Natural Science Foundation of China for Distinguished Young Scientists through Grant No. 51325103. J.A. Aguiar acknowledges support in part by Oak Ridge National Laboratory's Center User Facility, which is sponsored by the Scientific User Facilities Division, Office of Basic Energy Sciences, U.S. Department of Energy. F. Liu acknowledges the financial support from DOE-BES (No. DE-FG02-04ER46148).

\section{Appendix A. Supplementary material}




\section{References}

[1] J. Israelachvili, R. Pashley, The hydrophobic interaction is long range, decaying exponentially with distance, Nature 300 (1982) 341-342.

[2] D. Chandler, Interfaces and the driving force of hydrophobic assembly, Nature 437 (2005) 640-647.

[3] A. Tuteja, W. Choi, M.L. Ma, J.M. Mabry, S.A. Mazzella, G.C. Rutledge, G.H. McKinley, R.E. Cohen, Designing Superoleophobic Surfaces, Science 318 (2007) 1618-1622.

[4] X.C. Gui, H.B. Li, K.L. Wang, J.Q. Wei, Y. Jia, Z. Li, L.L. Fan, A.Y. Cao, H.W. Zhu, D.H. Wu, Recyclable carbon nanotube sponges for oil absorption, Acta Mater. 59 (2011) 4798-4804.

[5] R. Zhou, X. Huang, C.J. Margulis, B.J. Berne, Hydrophobic Collapse in Multidomain Protein Folding, Science 305 (2004) 1605-1609.

[6] A. Poynor, L. Hong, I.K. Robinson, S. Granick, Z. Zhang, P.A. Fenter, How Water Meets a Hydrophobic Surface, Phys. Rev. Lett. 97 (2006) 266101.

[7] U. Schmidt, G. Guigas, M. Weiss, Cluster Formation of Transmembrane Proteins Due to Hydrophobic Mismatching, Phys. Rev. Lett. 101 (2008) 128104.

[8] S. Kim, G.L. Haller, Solid electrolyte aided studies of NO-CO reaction on Pd, Solid State Ionics 136 (2000) 693-697.

[9] H. Yoshida, Y. Yazawa, T. Hattori, Effects of support and additive on oxidation state and activity of Pt catalyst in propane combustion, Catal. Today 87 (2003) 19-28.

[10] B.K. Rao, P. Jena, M. Manninen, R.M. Nieminen, Spontaneous fragmentation of multiply charged metal clusters, Phys. Rev. Lett. 58 (1987) 1188-1191.

[11] M. Brack, The physics of simple metal clusters: self-consistnt jellium model and semiclassical approaches, Rev. Mod. Phys. 65 (1993) 677-732.

[12] Emsley J. The Elements, 3rd edition. Oxford: Clarendon Press, 1998.

[13] J.K. Nørskov, N.D. Lang, Effective-medium theory of chemical binding: Application to chemisorptions, Phys. Rev. B 21 (1980) 2131-2136.

[14] M.J. Stott, E. Zaremba, Quasiatoms: An approach to atoms in nonuniform electronic systems, Phys. Rev. B 22 (1980) 1564-1583.

[15] M.S. Daw, M.I. Baskes, Embedded-atom method: Derivation and application to impurities, surfaces, and other defects in metals, Phys. Rev. B 29 (1984) 6443.

[16] G. Kresse, J. Furthmüller, Efficient iterative schemes for ab initio total-energy calculations using a plane-wave basis set, Phys. Rev. B 54 (1996) 11169-11186.

[17] G. Kresse, J. Hafner, Ab initio molecular dynamics for liquid metals. Phys. Rev. B 47 (1993) 558-561.

[18] J.P. Perdew, Y. Wang, Accurate and simple analytic representation of the electron-gas correlation energy, Phys. Rev. B 45 (1992) 13244-13249.

[19] P.E. Blöchl, Projector augmented-wave method, Phys. Rev. B 50 (1994) 17953-17979. 
[20] H.J. Monkhorst, J.D. Pack, Special points for Brillouin-zone integrations, Phys. Rev. B 13 (1976) 5188-5192.

[21] M.J. Puska, R.M. Nieminen, M. Manninen, Atoms embedded in an electron gas: Immersion energies, Phys. Rev. B 24 (1981) 3037-3047.

[22] C. Templier, R.J. Gaboriaud, H. Garem, Precipitation of Implanted Xenon in Aluminium, Mater. Sci. Eng. 69 (1985) 63-66.

[23] C.S. Becquart, C. Domain, Migration Energy of He in W Revisited by Ab Initio Calculations, Phys. Rev. Lett. 97 (2006) 196402.

[24] T. Seletskaia, Y. Osetsky, R.E. Stoller, G.M. Stocks, First-principles theory of the energetics of He defects in bcc transition metals, Phys. Rev. B 78 (2008) 134103.

[25] X.T. Zu, L. Yang, F. Gao, S.M. Peng, H.L. Heinisch, X.G. Long, R.J. Kurtz, Properties of helium defects in bcc and fcc metals investigated with density functional theory, Phys. Rev. B 80 (2009) 054104.

[26] H.B. Zhou, Y.L. Liu, S. Jin, Y. Zhang, G.N. Luo, G.H. Lu, Towards suppressing H blistering by investigating the physical origin of the H-He interaction in W, Nucl. Fusion 50 (2010) 115010.

[27] L. Yang, F. Gao, R.J. Kurtz, X.T. Zu, Atomistic simulations of helium clustering and grain boundary reconstruction in alpha-iron, Acta Mater. 82 (2015) 275-286.

[28] Y.L. Liu, Y. Zhang, H.B. Zhou, G.H. Lu, F. Liu, G.N. Luo, Vacancy trapping mechanism for hydrogen bubble formation in metal, Phys. Rev. B 79 (2009) 172103.

[29] K. Ohsawa, K. Eguchi, H. Watanabe, M. Yamaguchi, M. Yagi, Configuration and binding energy of multiple hydrogen atoms trapped in monovacancy in bcc transition metals, Phys. Rev. B 85 (2012) 094102.

[30] N. Fernandez, D. Kato, Hydrogen diffusion and vacancies formation in tungsten: Density Functional Theory calculations and statistical models, Acta Mater 94 (2015) 307-318.

[31] X.S. Kong, S. Wang, X.B. Wu, Y.W. You, C.S. Liu, Q.F. Fang, J.L. Chen, G.N. Luo, First-principles calculations of hydrogen solution and diffusion in tungsten: Temperature and defect-trapping effects, Acta Mater. 84 (2015) 426-435.

[32] F. Liu, B.K. Rao, S.N. Khanna, P. Jena, Nature of short range interaction between deuterium atoms in Pd, Solid State Commun. 72 (1989) 891-894.

[33] T. Seletskaia, Y. Osetsky, R.E. Stoller, G.M. Stocks, Magnetic Interactions Influence the Properties of Helium Defects in Iron, Phys. Rev. Lett. 94 (2005) 046403.

[34] K.O.E. Henriksson, K. Nordlund, A. Krasheninnikov, J. Keinonen, Difference in formation of hydrogen and helium clusters in tungsten, Appl. Phys. Let. 87 (2005) 163113.

[35] T. Tamura, R. Kobayashi, S. Ogata, A. M. Ito, First-principles investigation of possible clustering of noble gas atoms implanted in bcc tungsten, Modelling Simul. Mater. Sci. Eng. 22 (2014) 015002.

[36] D. Nishijima, M.Y. Ye, N. Ohno, S. Takamura, Incident ion energy dependence of bubble formation on tungsten surface with low energy and high flux helium plasma irradiation, J. Nucl. Mater. 313 (2003) 97-101.

[37] P.E. Lhuillier, T. Belhabib, P. Desgardin, B. Courtois, T. Sauvage, M.F. Barthe, A.L. Thomann, P. Brault, Y. Tessier, Helium retention and early stages of helium-vacancy complexes formation in low energy heliumimplanted tungsten, J. Nucl. Mater. 433 (2013) 305-313. 
[38] J.H. Evans, A. Van Veen, L.M. Caspers, Formation of helium platelets in molybdenum, Nature 291 (1981) 310312.

[39] J. Chen, W. Hoffelner, H. Ullmaier, Dislocation loops and bubbles in oxide dispersion strengthened ferritic steel after helium implantation under stress, Acta Mater. 56 (2008) 250-258.

[40] D. Brimbal, B. Décamps, J. Henry, E. Meslin, A. Barbu, Single- and dual-beam in situ irradiations of highpurity iron in a transmission electron microscope: Effects of heavy ion irradiation and helium injection, Acta Mater. 64 (2014) 391-401.

[41] A. Luukkainen, J. Keinonen, M. Erola, Density and size of neon bubbles in molybdenum, tantalum, and tantalum oxide, Phys. Rev. B 32 (1985) 4814-4817.

[42] A.V. Felde, J. Fink, Th. Muller-Heinzerling, J. Pfluger, B. Scheerer, G. Linker, Pressure of Neon, Argon, and Xenon Bubbles in Aluminum, Phys. Rev. Lett. 53 (1984) 922-925.

[43] R.J. Cox, P.J. Goodhew, J.H. Evans, A study of the solidification of argon bubbles in aluminium, Acta Metall. 35 (1987) 2497-2501.

[44] C. Templier, J.C. Desoyer, H. Garem, J.P. Riviere, Rhombohedron structure of xenon bubbles in Xe implanted Al, Acta Metall. 37 (1989) 933-940.

[45] R.P. Doerner, M.J. Baldwin, D. Nishijima, J. Roth, K. Schmid, Impact of beryllium surface layers on deuterium retention in tungsten, J. Nucl. Mater. 415 (2011) S717-S720.

[46] C. Domain, C.S. Becquart, J. Foct, Ab initio study of foreign interstitial atom $(\mathrm{C}, \mathrm{N})$ interactions with intrinsic point defects in $\alpha$-Fe, Phys. Rev. B 69 (2004) 144112.

[47] W. Ming, Z.Z. Fang, F. Liu, Effects of Li doping on H-diffusion in $\mathrm{MgH}_{2}$ : A first-principles study. J. Appl. Phys. 114 (2013) 243502. 


\section{Figure captions:}

Fig. 1. The plot shows the solution energy of a $\mathrm{He}$ atom and a $\mathrm{H}$ atom in a homogeneous electron gas as a function of the electron density.

Fig. 2. (a) Schematics of a hard-wall potential between an electrophobic solute and its surrounding electron medium and a close-packed spherical cluster of $\mathrm{N}$ atoms (inset). (b) TEM image of a Xe cluster formed from Xe implantation in Al, showing a close-packed crystalline structure. The [111] direction refers to out-of-plane direction for the Xe bubble, whose Fourier transform is shown in the inset. (c) and (d) The clustering energy of a He and Be cluster due to electrophobic interaction in a homogeneous electron gas of $0.08 \mathrm{e} / \AA^{3}$ electron density, respectively, following a universal scaling relation with the cluster size, $E_{\mathrm{c}} \propto\left(N^{2 / 3}-N\right)$ according to the hard-sphere model. The solid lines are fits of the calculated data using the theoretical model.

Fig. 3. The $d$-projected DOS for the nearest-neighboring $\mathrm{W}$ atom of interstitial He (a) and $\mathrm{H}$ (b), respectively, the $p$-projected DOS for He (a), and the $s$-projected DOS for H(b).The solid and dashed lines show the partial DOS of $\mathrm{W}, \mathrm{He}$, and $\mathrm{H}$ with a $\mathrm{He} / \mathrm{H}$ atom at respective TIS and OIS.

Fig. 4. The changes in electron density of $\mathrm{W}$ induced by interstitial He (a, b) and $\mathrm{H}(\mathrm{c}, \mathrm{d})$ occupying tetrahedral and octahedral sites.

Fig. 5. (a) Schematics of five dispersed TIS He atoms in W. (b) Schematics of the five-atom He cluster in W, obtained from the first-principles calculations. (c) and (d) The clustering energy of a $\mathrm{He}$ and $\mathrm{Be}$ cluster due to electrophobic interaction in $\mathrm{W}$, respectively, following a universal scaling relation with the cluster size, $E_{\mathrm{c}} \propto\left(N^{2 / 3}-N\right)$ according to the hard-sphere model. The labels are the same as in Fig. 2.

Fig. 6. The clustering energy of a Xe cluster due to electrophobic interaction in Al follows a universal scaling relation with the cluster size, $E_{\mathrm{c}} \propto\left(N^{2 / 3}-N\right)$ according to the hard-sphere model. The solid line is fit of the calculated data using the theoretical model. 
Fig. 7. The clustering energy of a He cluster due to electrophobic interaction in a W vacancy, following a universal scaling relation with the cluster size, $E_{\mathrm{c}} \propto\left(N^{2 / 3}-N\right)$ according to the hardsphere model. The solid line is fit of the calculated data using the theoretical model.

Fig. 8. A new design of radiation tolerant material. Schematics of the proposed design of a layered radiation protection shell based on electrophobic interaction. 


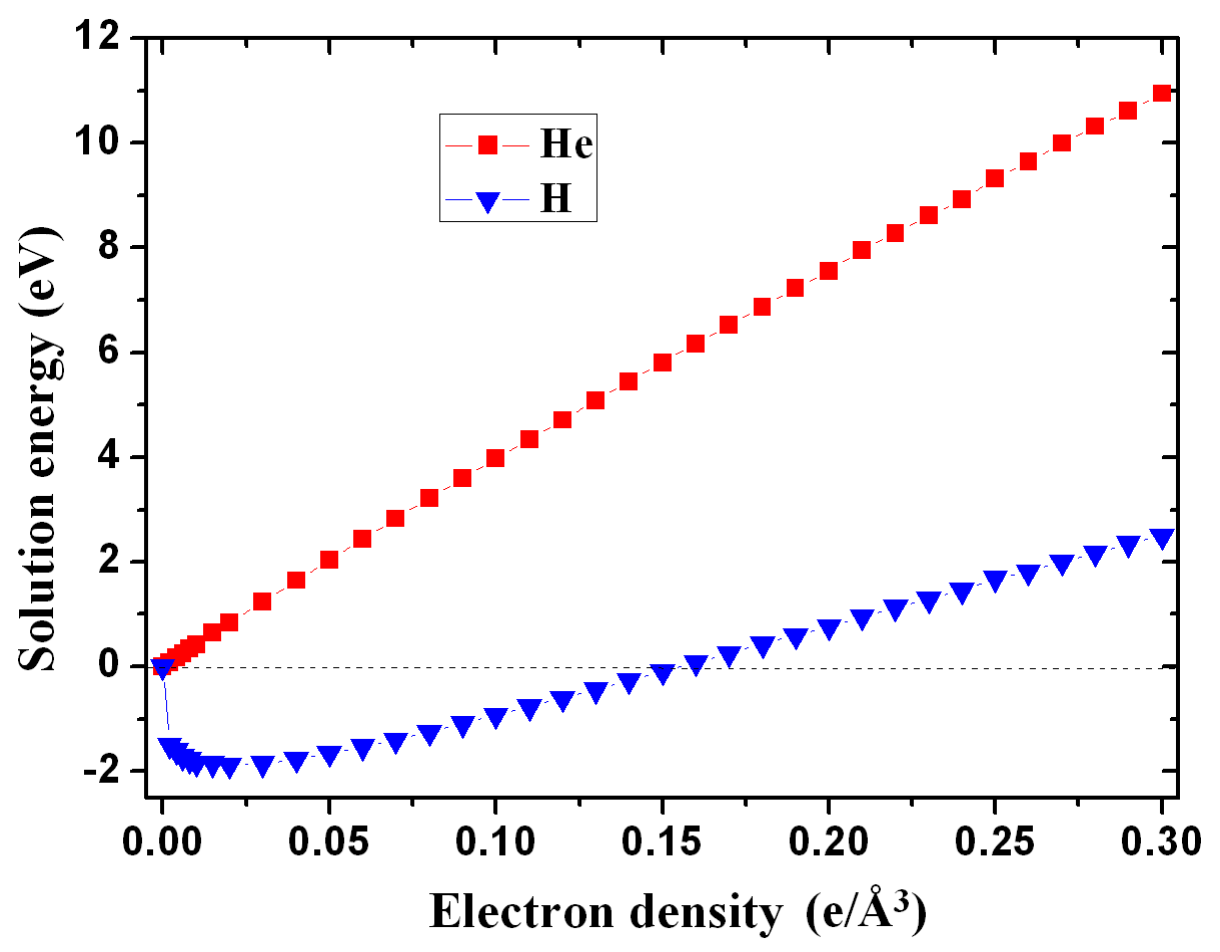

Fig. 1. 

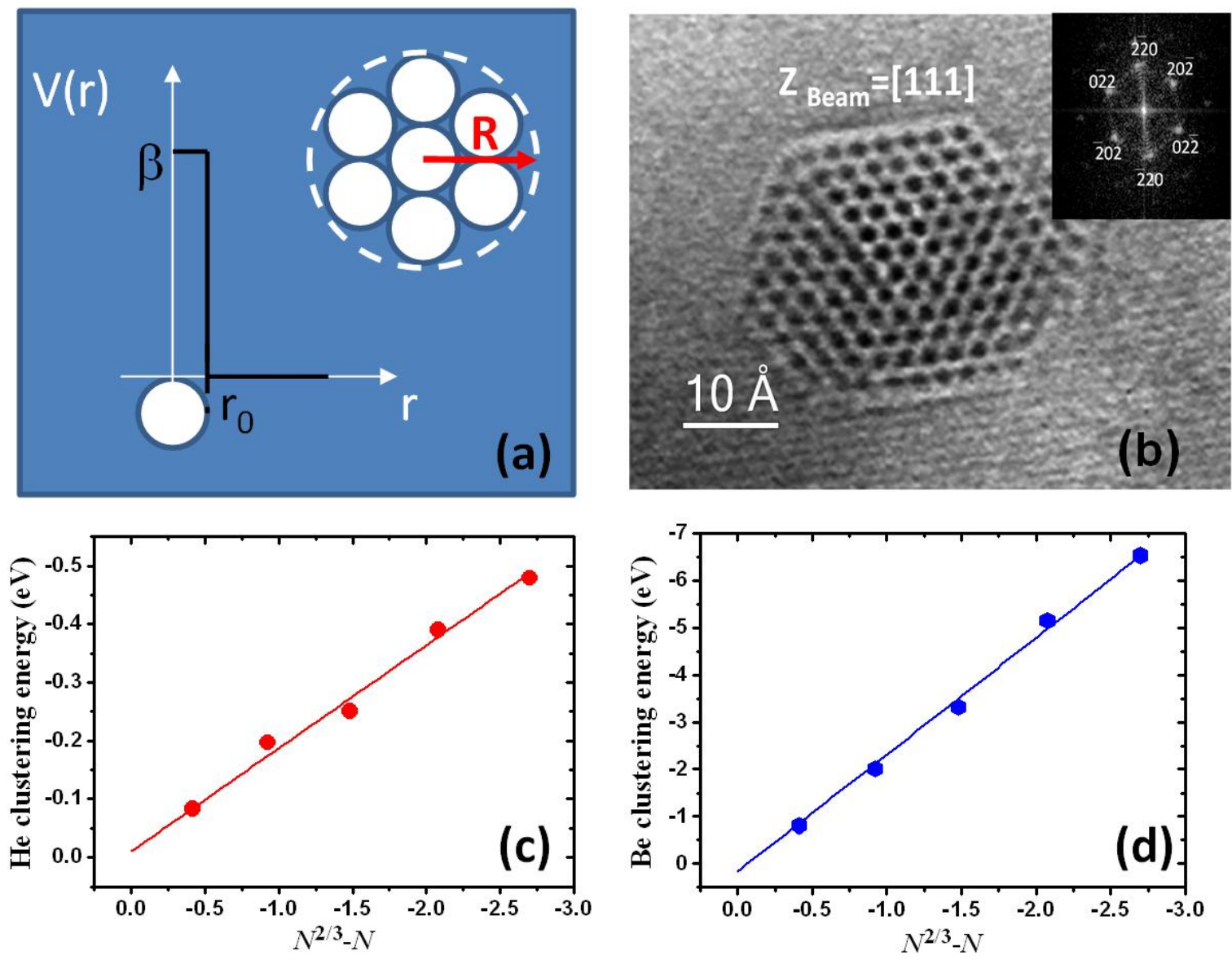

Fig. 2. 


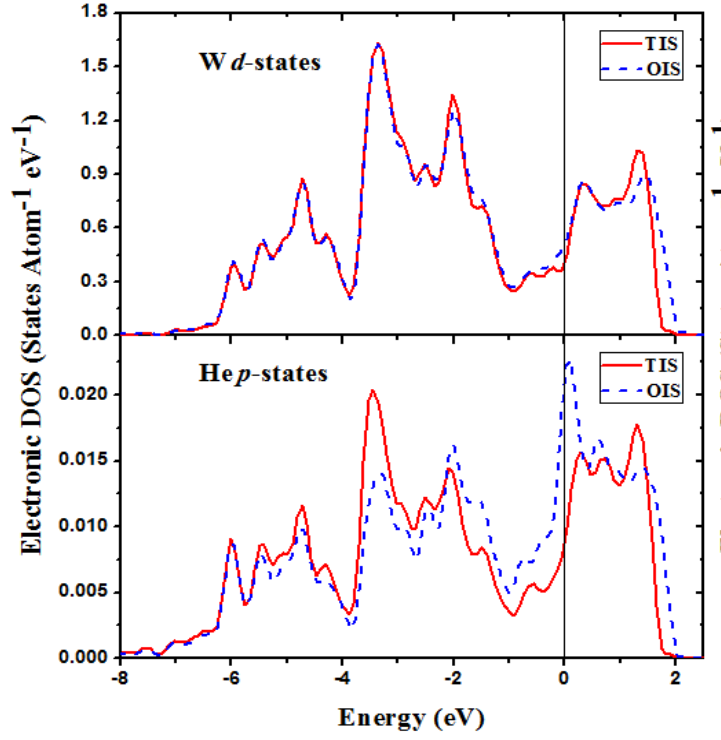

(a)

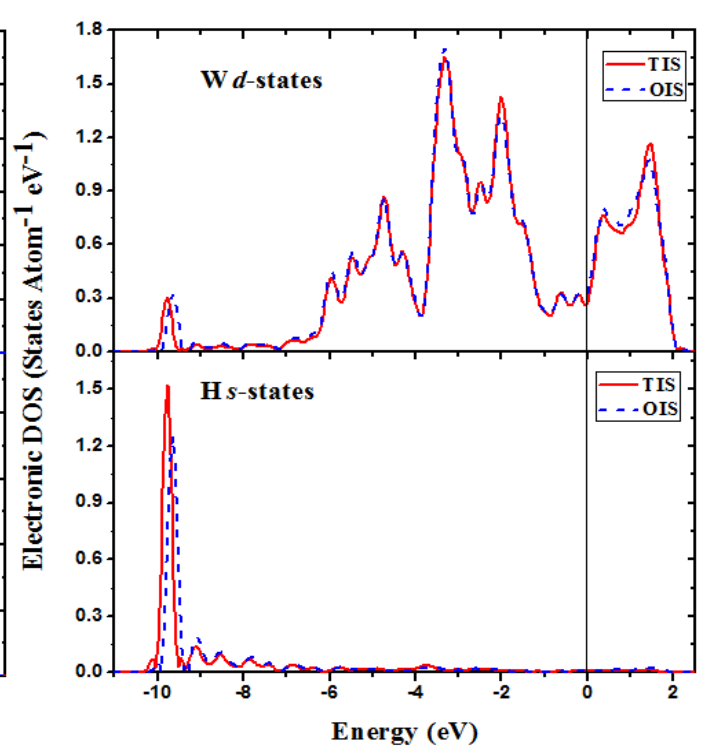

(b)

Fig. 3. 

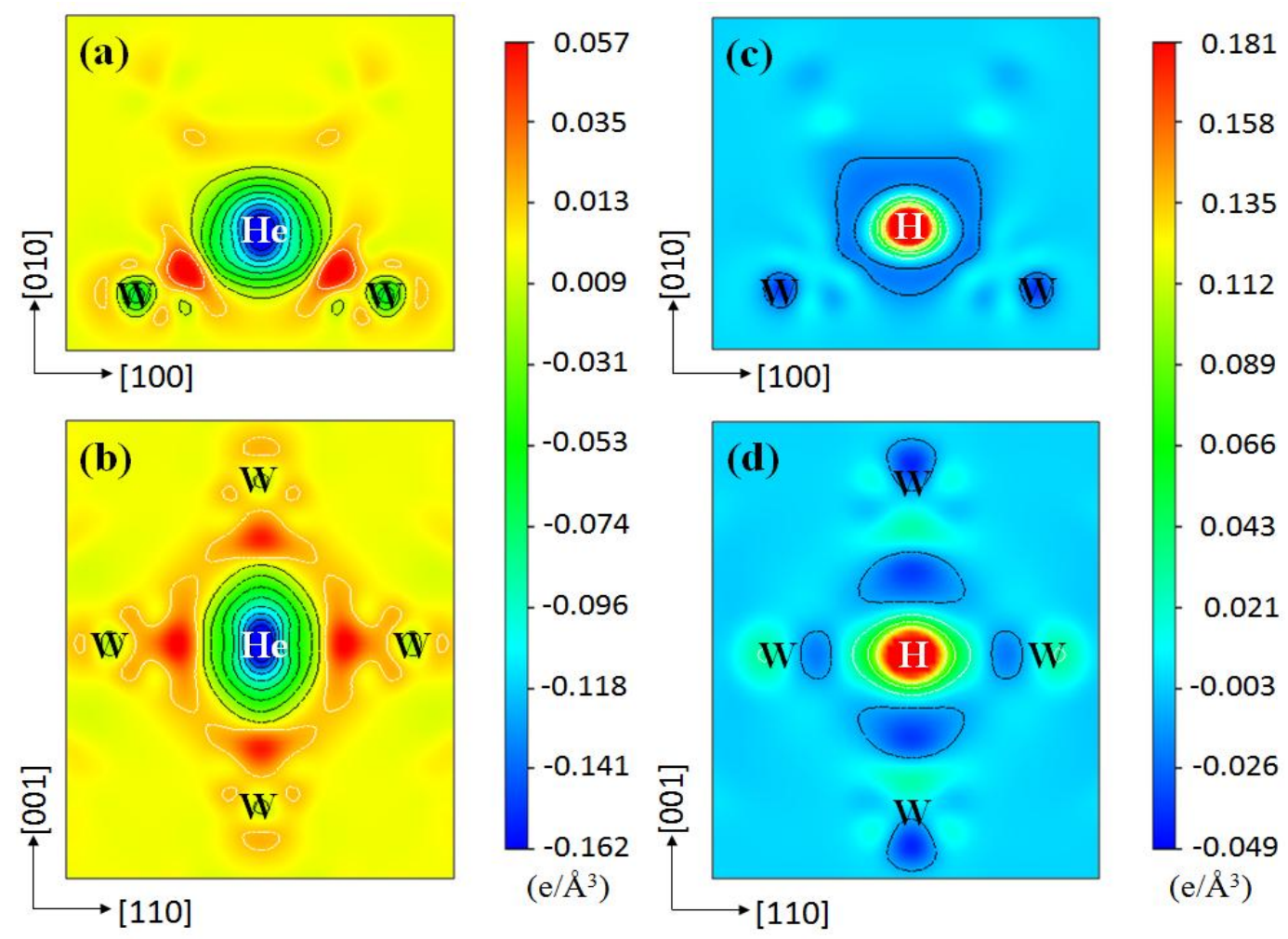

Fig. 4. 

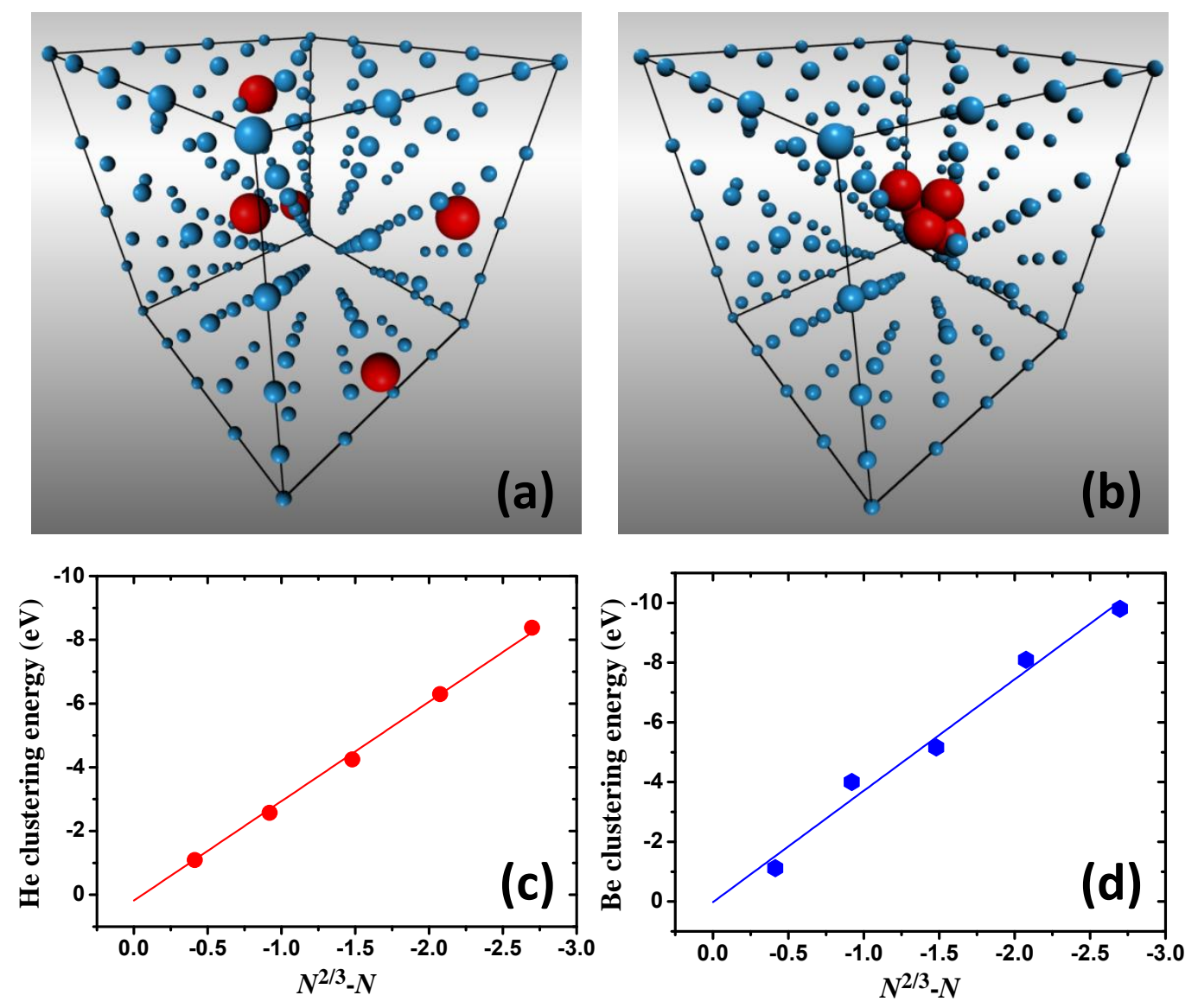

Fig. 5. 


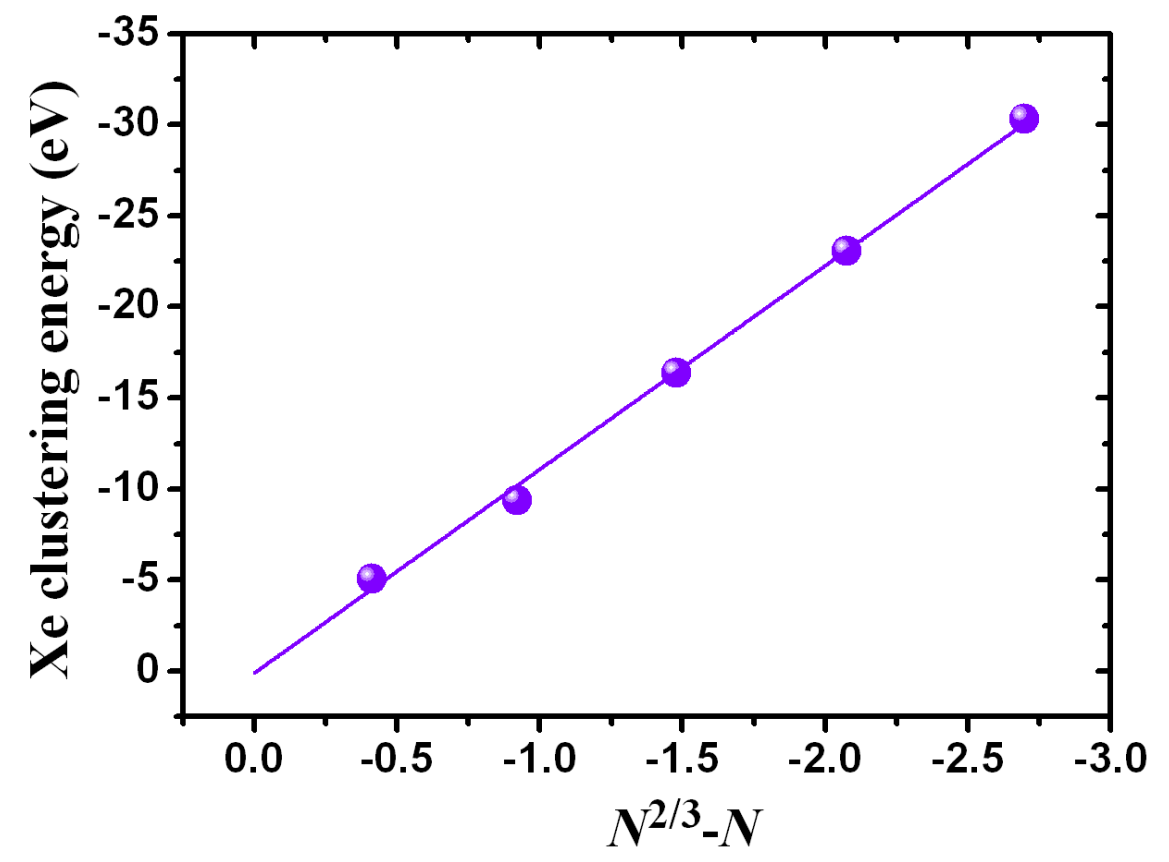

Fig. 6. 


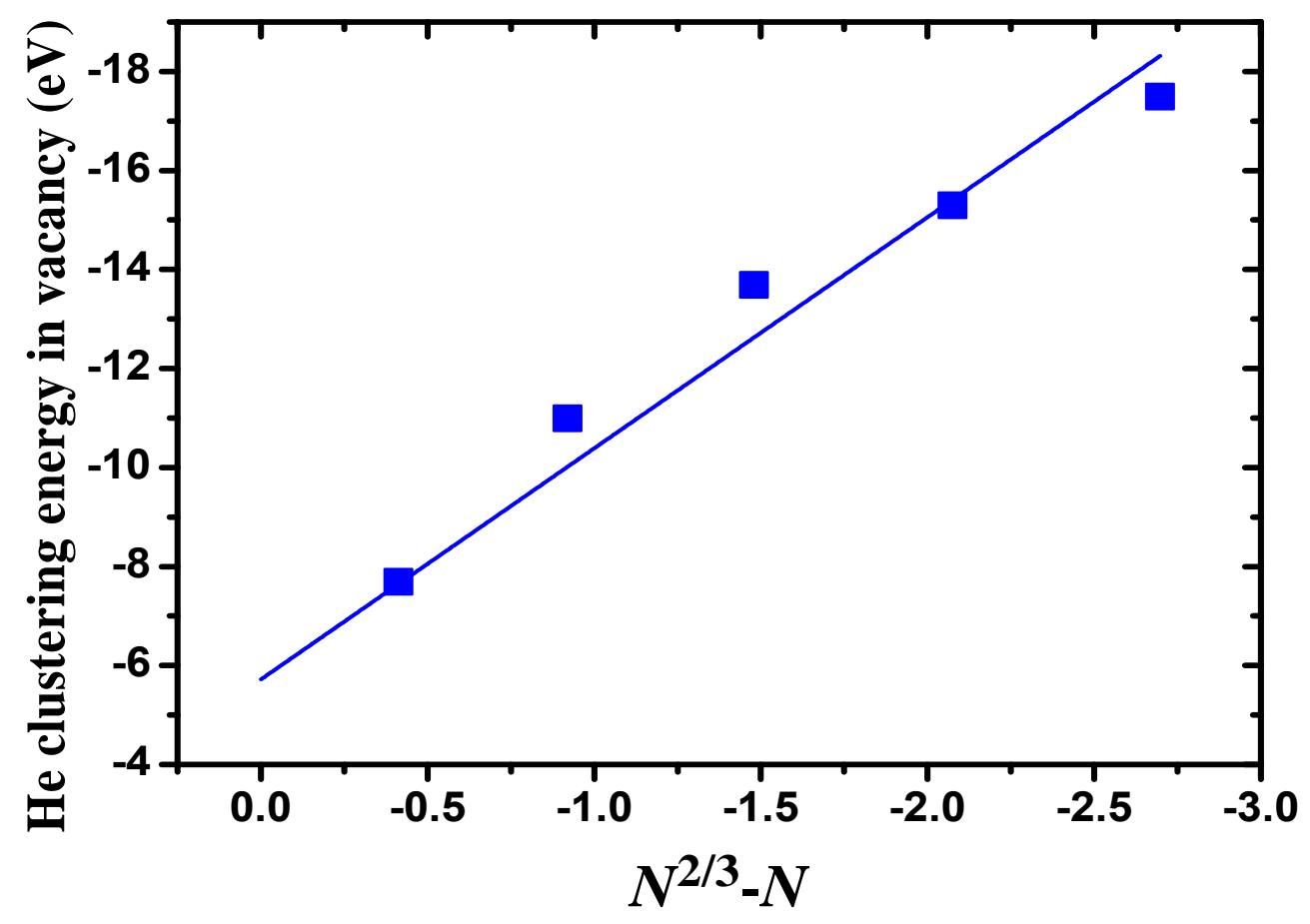

Fig. 7. 


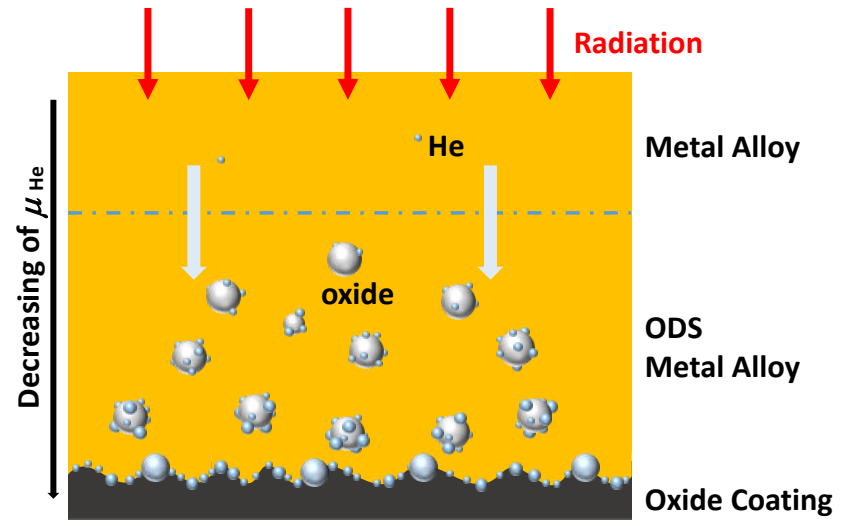

Fig. 8. 
Table 1

Demonstration of dominant contribution of electronic solution energy to the total solution energy of $\mathrm{He}$ in $\mathrm{W}$ at three different sites TIS, OIS and SS. All energies are in units of $\mathrm{eV}$ and electron density is in units of $\mathrm{e} / \AA^{3}$.

\begin{tabular}{cccc}
\hline & TIS & OIS & SS \\
\hline $\begin{array}{c}\text { Total solution energy } \\
\text { Electron density }\end{array}$ & 6.18 & 6.39 & 1.59 \\
$\begin{array}{c}\text { Electronic solution energy } \\
\text { (LDA) }\end{array}$ & 0.171 & 0.162 & 0.026 \\
$\begin{array}{c}\text { Electronic solution energy } \\
\text { (Derived) }\end{array}$ & 5.18 & 5.24 & 1.56 \\
$\begin{array}{c}\text { He-induced deformation energy } \\
\text { (D) }\end{array}$ & 1.00 & 1.15 & 0.03 \\
\hline
\end{tabular}




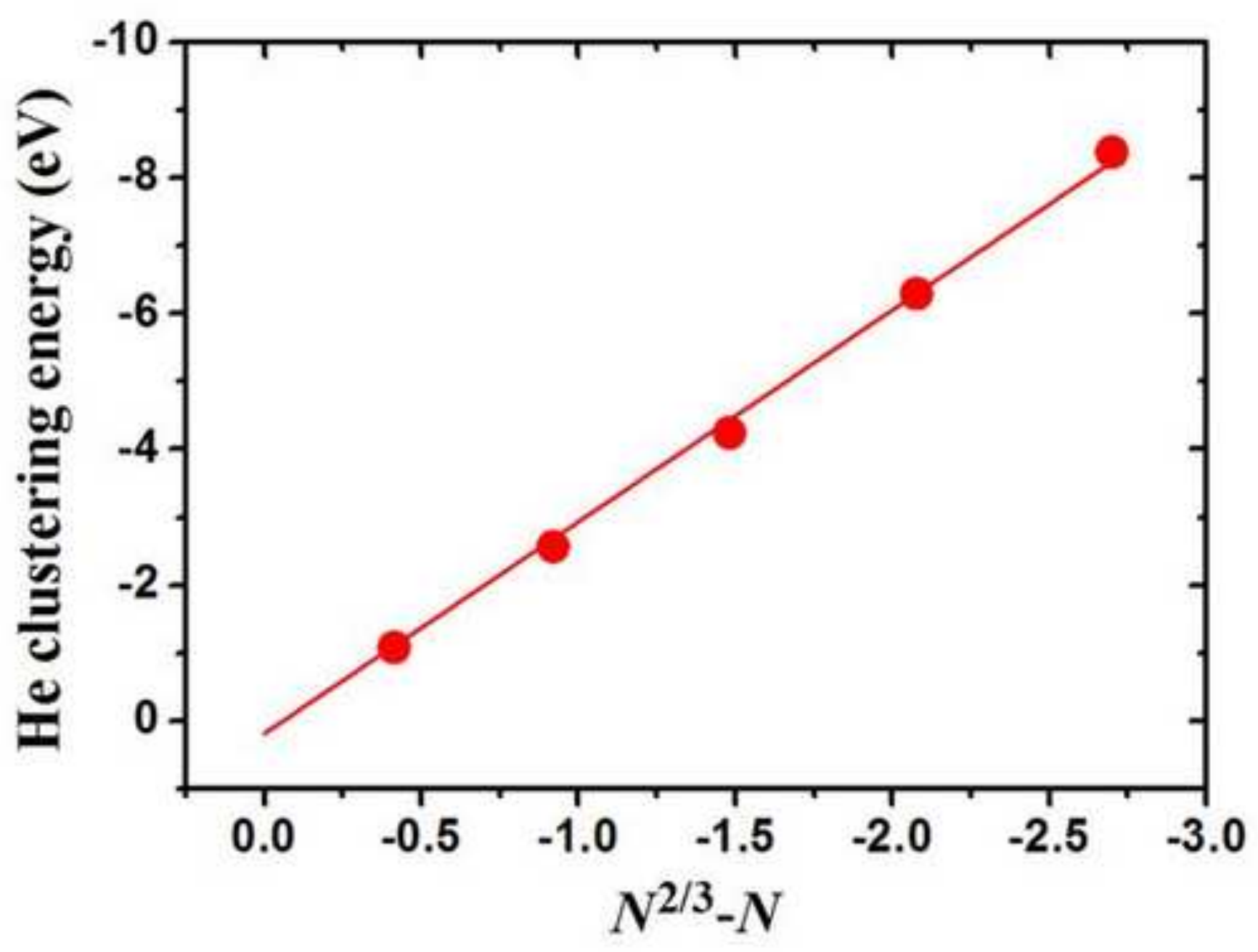

The clustering energy of a He cluster in W

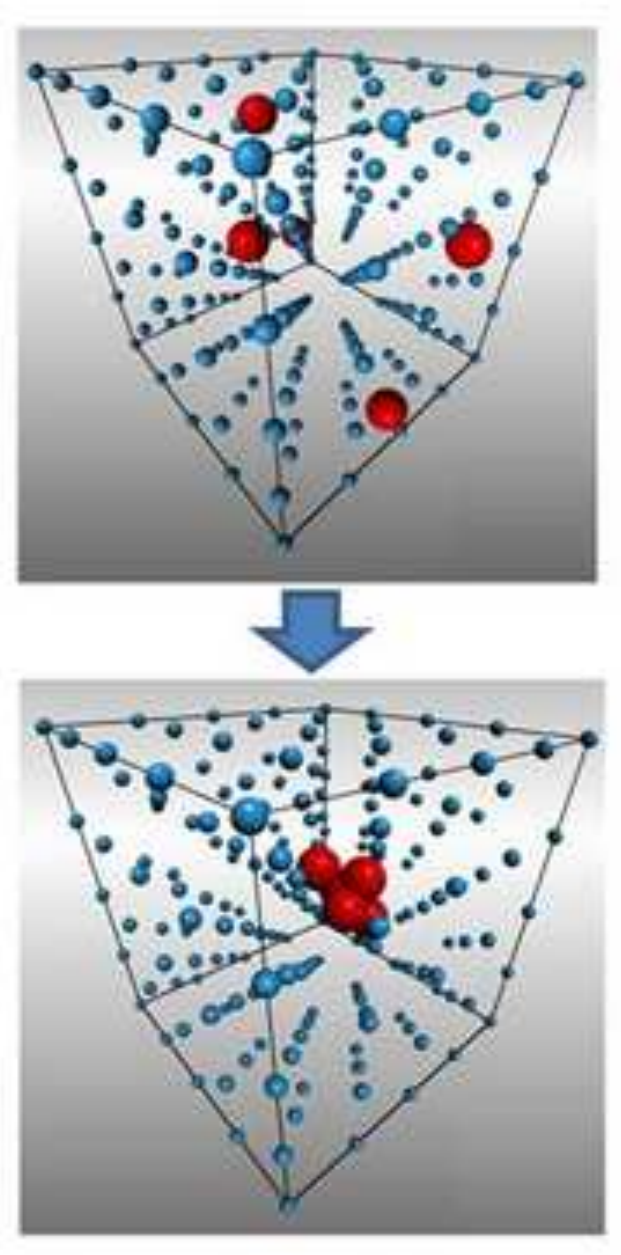

Schematics of the fiveatom He cluster in W 